\title{
Discrepancies of SARS-CoV-2 testing results among patients with total laryngectomy
}

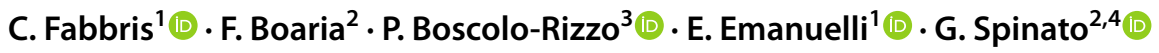

Received: 19 July 2021 / Accepted: 24 November 2021 / Published online: 2 December 2021

(c) The Author(s), under exclusive licence to Springer-Verlag GmbH Germany, part of Springer Nature 2021

\begin{abstract}
Purpose Prevention of severe acute respiratory syndrome coronavirus 2 (SARS-CoV-2) infection is obtained with nasopharyngeal swabs. By the way, there is no consensus regarding sampling in totally laryngectomized subjects (who thus breathe directly by the tracheostomy and, theoretically, may be infected in the trachea). The aim of this study is to evaluate possible differences between swab results in the trachea and in the nasopharynx of this category of patients.

Methods A retrospective chart review was performed in April 2021 among patients who previously had been operated on for total laryngectomy and who underwent swabs for SARS-CoV-2 research in 3 health-care centers in Northern-Eastern Italy. Data regarding the site of swabbing (trachea or nasopharynx) were analyzed. A comprehensive review of the literature regarding the same topic was then performed.

Results A total of 25 totally laryngectomized subjects underwent swabs. Among them, 5 tested positive in the trachea (1) and in the nasopharynx (4). According to the literature review, 4 more subjects tested positive in the trachea (1) and in the nasopharynx (3). Data were overall divergent and no statistically significant correlations emerged between results of the tests performed in the two sites.

Conclusion Due to these discrepancies, both tracheal and nasopharyngeal swabs are recommended in these kinds of patients, to obtain a reliable test and to avoid false negatives.
\end{abstract}

Keywords Total laryngectomy $\cdot$ Nasopharyngeal swab $\cdot$ Tracheal swab $\cdot$ SARS-CoV-2 $\cdot$ COVID-19

\section{Introduction}

The coronavirus disease (COVID-19) pandemic spread out since December 2019. To obtain an early diagnosis of infection by the severe acute respiratory syndrome coronavirus 2 (SARS-CoV-2), international guidelines recommend proper testing [1]. To date, nasopharyngeal swabbing is the gold

C. Fabbris

cristoforo.fabbris@gmail.com

1 Unit of Otolaryngology, University Hospital of Treviso, Treviso, Italy

2 Section of Otolaryngology and Regional Centre for Head and Neck Cancer, Department of Neurosciences, University of Padova, Treviso, Italy

3 Section of Otorhinolaryngology, Azienda Sanitaria Universitaria Integrata di Trieste, Trieste, Italy

4 Section of Oncology and Immunology, Department of Surgery, Oncology and Gastroenterology, University of Padova, Padova, Italy standard method to obtain those specimens [2-4]. According to the guidelines, specimens obtained from the upper airways are adequate especially for asymptomatic or mild cases.

On the other hand, lower respiratory specimens are advised if collected later in the course of COVID-19 or in patients with a negative upper respiratory tract sampling and with a strong clinical suspicion of the disease [5]. Other sampling alternatives, such as combined oropharyngeal and nares/nasal swab, middle turbinate or tongue swab, have been developed, but to date, they have not been validated [6].

Currently, there are no guidelines regarding subjects who previously underwent total laryngectomy. In these cases, patients directly breathe through the tracheostoma, so the airflow bypasses nose and mouth. Thus, the execution of diagnostic tests must take into consideration the abovementioned anatomical alterations of the upper airways, since the nasopharynx and oral cavity are separated from the lower airways. To date, different methods have been suggested, and generally both nasal and tracheal testing have been 
recommended, since discrepancies have been encountered between samples collected from the two areas [7-10]. By the way, only case reports have been presented; thus, no robust conclusions have been obtained [8-10].

The aim of the present study is to present results of swab tests among a case series of patients with total laryngectomy and to perform a review of the literature, to evaluate the most appropriate testing method.

\section{Methods}

In April 2021, a retrospective analysis of charts among patients who previously underwent total laryngectomy has been performed. Data about the patients were collected from 3 health care centers in the same geographical area (Treviso, Mestre and Mirano hospitals, Italy). An initial screening was performed by considering whether they had or had not underwent a SARS-CoV-2 test. Subsequently, among those who underwent swabbing, the following aspects have been analyzed: anatomical site where the sample was collected, presence or absence of speech prosthesis (SP), presence or absence of symptoms related to SARS-CoV-2 infection, type of performed test analysis (molecular or antigenic).

A comprehensive review of the literature on the topic has been performed. Statistical analysis (Fisher's exact test) was performed on data extracted from the spreadsheet.

All procedures described in the present paper followed the ethical principles of the Declaration of Helsinki.

\section{Results}

\section{Retrospective analysis}

Overall, 74 charts have been reviewed, belonging to 60 men and 14 women. Twenty-five patients underwent nasopharyngeal swab (NP) once or more times for SARS-CoV-2 research due to presence of symptoms (such as fever), to contact with positive patients, or for screening reasons. The swabs were analyzed with Real-Time Polymerase Chain Reaction (13 cases), Antigenic Test (5), or both (7). Among the patients, 6 have been double-tested both in the nasopharynx and through the tracheostoma, with two different swabs and consequently two distinct analyses.

A total of 5 patients resulted to be infected by SARSCoV-2. Four patients tested positive with NP. Two subjects were only tested in the nasopharynx, while other 2 were tested both in the nasopharynx and the trachea. The latter 2 patients tested negative on the tracheal swab (TR): one of them, who had SP, was found to be positive with NP but then he tested negative at TR; the other patient, who had not SP, after testing positive with multiple NPs, was tested also with TR which resulted negative. One patient underwent multiple NPs which tested negative and subsequent TR resulted to be positive; the patient had the SP, but it was found to be closed by overgrown mucosa on the esophageal side.

No statistically significant differences were present between results of NP and of TR $(p>0.05)$. Patients' characteristics are summarized in Supplementary Table 1.

\section{Review of the literature}

A total of 10 titles and abstracts were retrieved. Among them, 7 were excluded due to lack of clinical cases, or since they were clearly not related to the object of research [7, 11-16]. Eventually, 3 articles dealing about 4 patients were analyzed [8-10]. Patients underwent only NP in 2 cases, while both NP and TR were performed in the other 2 cases. $\mathrm{NP}$ tested positive in 3 cases. TR tested negative in 1 patient who had a positive NP, and it tested positive in 1 patient who resulted to be negative within the nasopharynx. SP was present in 1 subject who underwent NP only and in another patient who underwent double-testing. Table 1 summarizes characteristics of both the included positive patients of our study and the patients of the literature review. By considering patients who were double-tested, no statistically significant differences resulted between the results of NPs and TRs $(p>0.05)$. No correlation was even present between symptoms and the presence of a SP $(p>0.05)$.

Table 1 Summary of positive cases obtained both from the retrospective chart review (patients 1, 7, 11, 13, 17) and from the literature review (patients A, B, C, D)

\begin{tabular}{|c|c|c|c|c|c|c|c|c|c|}
\hline \multirow[b]{2}{*}{ Patient no } & \multicolumn{5}{|c|}{ Chart review patients } & \multicolumn{4}{|c|}{ Literature review patients } \\
\hline & 1 & 7 & 11 & 13 & 17 & $\mathrm{~A}^{8}$ & $\mathrm{~B}^{8}$ & $\mathrm{C}^{9}$ & $\mathrm{D}^{10}$ \\
\hline NP & Neg & Pos & Pos & Pos & Pos & Pos & Pos & Pos & Neg \\
\hline $\mathrm{TR}$ & Pos & Not performed & Neg & Not performed & Neg & Not performed & Not performed & Neg & Pos \\
\hline SP & Y, closed & $\mathrm{Y}$ & $\mathrm{Y}$ & $\mathrm{N}$ & $\mathrm{N}$ & $\mathrm{Y}$ & $\mathrm{N}$ & $\mathrm{Y}$ & $\mathrm{N}$ \\
\hline Symptoms & $\mathrm{Y}$ & $\mathrm{Y}$ & $\mathrm{Y}$ & $\mathrm{N}$ & $\mathrm{N}$ & $\mathrm{Y}$ & $\mathrm{Y}$ & $\mathrm{N}$ & $\mathrm{Y}$ \\
\hline
\end{tabular}

$N P$ (nasopharyngeal swab), TR (tracheal swab), SP (speech prosthesis), $Y$ (yes), $N$ (no), Neg (negative), Pos (positive). Superscript numbers refer to References' list 


\section{Discussion}

According to the present study, there was no correlation between results of swabs performed in the nasopharynx and those performed through the tracheostoma among patients with total laryngectomy.

Correct procedure of collecting a sample for SARS$\mathrm{CoV}-2$ testing in this type of patients is not clearly specified in the international guidelines. The matter of whether a laryngectomized subject should be tested via a nasopharyngeal sample or via a tracheal one has been discussed only in a few papers $[7,9,10]$. Although totally laryngectomized patients have no significant airflow through the nasopharynx, they can still develop sinonasal diseases; therefore, testing should be performed both in the upper (nasopharynx) and lower (tracheal) areas [7].

Moreover, SP may theoretically represent a link between upper and lower airways [9]: pt. C resulted to be positive with NP and negative with TR, the SP was present and might have allowed propagation from the trachea to the nasopharynx, but on the other hand, this would not explain why the TR tested negative.

Even if it is a small case series, in our chart review, we found many discrepancies (Table 1). In particular, pt. 11 had a negative TR and a positive NP. This result, similarly to pt. C [9], is in contrast with the possible propagation mechanism from the trachea to the nasopharynx explained above. On the other hand, pt. 17 (who did not have SP) had a surprising negative TR and a positive NP. As stated by Schmid et al. [10], the primary site of infection could have been the lower respiratory tract, potentially followed by later infection of the upper respiratory one via autoinoculation or systemic infection. Therefore, these cases strengthen the recommendation of testing the patients with both nasopharyngeal and tracheal sample.

Certainly, the main limitation of the present study is the low number of included patients. Moreover, we do not have enough data to find a strict correlation between the presence of SP and its effect on the different virus colonization in trachea or nasopharynx. Nevertheless, we underline that disparate results can be found when testing these patients in different anatomical sites.

Different papers have been published so far regarding the importance of symptoms in early detection of COVID19 , especially alterations of sense of smell [17-20]. Since among laryngectomized patients, the nasal airflow is practically absent, other symptoms should be investigated (e.g., fever, dyspnea, fatigue...). In the whole present case series, no correlation emerged between symptoms and the presence of the SP, or the presence of a positive NP or TR. This underlines the independence of COVID-19 with the site of colonization by the virus. Since the SP seems not to always represent the communication between nasopharyngeal and tracheal colonization sites, double-testing is mandatory to avoid false negatives.

\section{Conclusion}

In case of performing swabs in patients with total laryngectomy, it is important not to test only one site. Among these patients, a negative test obtained in the nasopharynx or in the trachea does not imply that even the other site is negative. Moreover, the SP seems not to be related with contemporary colonization or non-colonization of the two sites. By considering our data and the data available in literature, it is strongly recommended to perform the swab in both the anatomical sites to possibly detect SARS-CoV-2.

Further results among a wider case series are certainly needed to better comprehend the mechanism of propagation of the virus and the possible correlation of swab results between nasopharynx and trachea.

Supplementary Information The online version contains supplementary material available at https://doi.org/10.1007/s00405-021-07203-8.

\section{Declarations}

Conflict of interest The authors have no conflict of interest, funding or financial relationships. An informed consent has been obtained for any procedure involving the patients described in this article. The manuscript has not been submitted to more than one journal for simultaneous consideration. The manuscript has not been published previously (party or in full). All of the authors have participated in the planning, writing or revising the manuscript.

\section{References}

1. Centers for Disease Control and Prevention. Interim Guidelines for Collecting, Handling, and Testing Clinical Specimens from Persons for Coronavirus Disease 2019 (COVID-19). https://www. cdc.gov/coronavirus/2019-ncov/lab/guidelines-clinical-speci mens.html. Accessed in 31 May 2021

2. Spinato G, Gaudioso P, Boscolo Rizzo P et al (2021) Risk management during COVID-19: safety procedures for otolaryngologists. Acta Biomed. https://doi.org/10.23750/abm.v92i1.11281 (Published 2021 Feb 16)

3. Fabbris C, Cestaro W, Menegaldo A et al (2021) Is oro/nasopharyngeal swab for SARS-CoV-2 detection a safe procedure? Complications observed among a case series of 4876 consecutive swabs. Am J Otolaryngol 42:102758. https://doi.org/10.1016/j. amjoto.2020.102758

4. Frezza D, Fabbris C, Franz L et al (2021) A SARS-CoV-2 detection method based on nasal and nasopharyngeal lavage fluid: a pilot feasibility study. Laryngoscope Investig Otolaryngol. https:// doi.org/10.1002/lio2.625

5. Wang W, Xu Y, Gao R, Lu R, Han K, Wu G, Tan W (2020) Detection of SARS-CoV-2 in different types of clinical specimens. JAMA 323:1843-1844. https://doi.org/10.1001/jama.2020.3786 
6. Spinato G, Fabbris C, Menegaldo A, Marciani S, Gaudioso P, Da Mosto MC, Frezza D, Boscolo-Rizzo P (2021) Correct execution of the nasopharyngeal swab: a fundamental method to improve diagnosis of SARSCoV-2 infection. J Doct Nurs Pract. https://doi. org/10.1891/JDNP-D-20-00040 (Epub ahead of print. PMID: 34108199)

7. Hennessy M, Bann DV, Patel VA, Saadi R, Krempl GA, Deschler DG, Goyal N, Choi KY (2020) Commentary on the management of total laryngectomy patients during the COVID-19 pandemic. Head Neck 42:1137-1143. https://doi.org/10.1002/hed.26183 (Epub 2020 Apr 23. PMID: 32298016; PMCID: PMC7262329)

8. Paderno A, Fior M, Berretti G, Del Bon F, Schreiber A, Grammatica A, Mattavelli D, Deganello A (2021) COVID-19 and total laryngectomy-a report of two cases. Ann Otol Rhinol Laryngol 130(1):104-107. https://doi.org/10.1177/0003489420935500 (Epub 2020 Jun 25 PMID: 32583671)

9. Patel TR, Teitcher JE, Tajudeen BA, Revenaugh PC (2020) Disparate nasopharyngeal and tracheal COVID-19 diagnostic test results in a patient with a total laryngectomy. Otolaryngol Head Neck Surg 163(4):710-711. https://doi.org/10.1177/0194599820 933605 (Epub 2020 Jun 9 PMID: 32513036)

10. Schmid J, Prattes J, Kaufmann-Bühler AK, Riedl JM (2021) Sampling site matters when testing for COVID-19 after total laryngectomy: a case report. Wien Klin Wochenschr 18:1-2. https://doi. org/10.1007/s00508-021-01826-x (Epub ahead of print. PMID: 33599848; PMCID: PMC7890779)

11. De Seta D, Marrosu V, Russo FY, Carta F, Puxeddu R (2020) Closed total laryngectomy during the COVID-19 pandemic disease. Laryngoscope 130(11):2622-2624. https://doi.org/10.1002/ lary. 28919

12. Longobardi Y, Galli J, D'Alatri L et al (2021) Patients with voice prosthesis rehabilitation during the COVID-19 Pandemic: analyzing the effectiveness of remote triage and management. Otolaryngol Head Neck Surg 164(2):277-284. https://doi.org/10.1177/ 0194599820948043

13. Goldstein DP, Ralph G, de Almeida JR et al (2020) Tracheoesophageal voice prosthesis management in laryngectomy patients during the COVID-19 pandemic. J Otolaryngol Head Neck Surg. https://doi.org/10.1186/s40463-020-00456-z (Published 2020 Aug 10)
14. Araújo AMB, Silva JMCD, Pernambuco L (2020) Specific care for total laryngectomized patients during the COVID-19 pandemic in the Brazilian reality. Cuidados específicos com laringectomizados totais durante a pandemia de COVID-19 na realidade brasileira. Codas. https://doi.org/10.1590/2317-1782/20192020167

15. Coleman H, Tikka T, Okhovat S, Kang SK (2020) Planning considerations prior to laryngectomy for a patient infected with severe acute respiratory syndrome coronavirus- 2 pre-operatively [published online ahead of print, 2020 Nov 4]. J Laryngol Otol. https:// doi.org/10.1017/S0022215120002388

16. Gallo O, Bruno C, Locatello LG et al (2021) The impact of the COVID-19 pandemic on the quality of life of head and neck cancer survivors. Support Care Cancer 29(11):6297-6304. https:// doi.org/10.1007/s00520-021-06198-6

17. Spinato G, Costantini G, Fabbris C et al (2021) The importance of early detection of ENT symptoms in mild-to-moderate COVID19. Acta Otorhinolaryngol Ital 41:101-107. https://doi.org/10. 14639/0392-100X-N1038

18. Boscolo-Rizzo P, Menegaldo A, Fabbris C et al (2021) Six-month psychophysical evaluation of olfactory dysfunction in patients with COVID-19. Chem Senses. https://doi.org/10.1093/chemse/ bjab006 (PMID: 33575808; PMCID: PMC7929204)

19. Boscolo-Rizzo P, Polesel J, Spinato G, Menegaldo A, Fabbris C, Calvanese L, Borsetto D, Hopkins C (2020) Predominance of an altered sense of smell or taste among long-lasting symptoms in patients with mildly symptomatic COVID-19. Rhinology 58(5):524-525. https://doi.org/10.4193/Rhin20.263 (PMID: 32683438)

20. Capriotti V, Mattioli F, Guida F et al (2021) COVID-19 in the tonsillectomised population. Acta Otorhinolaryngol Ital. https://doi. org/10.14639/0392-100X-N1436 (Epub ahead of print. PMID: 33970896)

Publisher's Note Springer Nature remains neutral with regard to jurisdictional claims in published maps and institutional affiliations. 